P1-411 USING MULTIPLE MEASURES OF SOCIAL INEQUALITIES TO STUDY TIME TRENDS IN SMOKING INEQUALITIES

doi:10.1136/jech.2011.142976g.1

R Charafeddine, ${ }^{*}$ S Demarest, J Van der Heyden, H V Oyen. Scientific Institute of Public Health, Brussels, Belgium

Introduction Trends in social inequalities in smoking have been examined in a number of international publications; however these studies have rarely used multiple measures of health inequalities and did not compare simultaneously results in terms of relative vs absolute inequalities and measures of total impact vs measure of effect.

Methods Data from four successive waves of the Belgian Health Interview Survey $(1997,2001,2004,2008)$ were used to study trends in educational inequalities in daily smoking and calculate 4 measures of inequalities: the slope index of inequality (SII), the relative index of inequality (RII), both accounting for the size of each educational group, the population attributable risk (PAR), and the OR comparing the two extreme education groups.

Results All examined measures pointed towards significant inequalities in smoking. Time trends in social inequalities in smoking differed according to the indicator used. Looking at the relative measures of inequality, we found a significant increase between 1997 and 2008 especially for females for the OR (test of trend $=+5 \% \quad p=0.02$ for females and trend $=+8 \% \quad p=0.08$ for males) and a weaker slope for the RII (test of trend $=+1 \%$ $p=0.08$ for females and trend $=+1 \% p=0.07$ for males). Looking at the absolute measures (PAR and SII), we found no significant increase.

Conclusion These results could be explained by the reduced prevalence of tobacco smoking in the country and the increase in the overall educational level of the population.

\section{P1-412 STABILITY OF BIOMARKERS IN PLASMA FROZEN AT VARIOUS TEMPERATURES FOR SEVERAL YEARS: IMPLICATIONS FOR PROSPECTIVE EPIDEMIOLOGICAL STUDIES}

doi:10.1136/jech.2011.142976g.2

\section{S Clark, ${ }^{*}$ M Hill, R Peto, R Collins. CTSU, University of Oxford, Oxford, UK}

Introduction Long-term storage of blood samples in large prospective epidemiological studies enables future biochemical measurements. However, samples may be stored for more than 10 years before analysis, and information is scarce on the long-term stability of biomarkers in frozen plasma. We have investigated the stability of six lipid analytes and creatinine in samples frozen at $-20^{\circ} \mathrm{C},-40^{\circ} \mathrm{C}$, $-80^{\circ} \mathrm{C}$ and, in nitrogen vapour, at $-150^{\circ} \mathrm{C}$ for 13 years.

Methods Multiple $1 \mathrm{ml}$ EDTA plasma aliquots were prepared from blood samples from 21 healthy individuals aged 25-60 years. One aliquot from each subject was analysed immediately for apolipoprotein A1, apolipoprotein B, directly-measured HDL-C and LDL-C, total cholesterol, triglycerides and creatinine. Remaining aliquots were stored at $-20^{\circ} \mathrm{C},-40^{\circ} \mathrm{C},-80^{\circ} \mathrm{C}$ or $-150^{\circ} \mathrm{C}$. An aliquot from each individual and each storage temperature was analysed at intervals over 13 years to provide direct comparisons between temperatures.

Results Compared with storage in nitrogen vapour, storage at $-80^{\circ} \mathrm{C}$ for up to 13 years resulted in a mean plasma concentration change of $<6 \%$ for all analytes except LDL which changed $<10 \%$. However, at higher storage temperatures several analytes changed markedly. For example, after 4 years of storage at $-20^{\circ} \mathrm{C}$, the mean plasma concentration of HDL-C decreased by more than $14 \%$ and LDL-C by more than $35 \%$.

Conclusion This study provides some of the most extensive evidence to date on long-term stability of important lipid analytes and creatinine in frozen plasma, and demonstrates the need to store plasma at $-80^{\circ} \mathrm{C}$ or below for long-term prospective studies.

\section{P1-413 FACTORS ASSOCIATED WITH DIETARY PATTERNS AMONG PREGNANT BRAZILIAN WOMEN}

doi:10.1136/jech.2011.142976g.3

${ }^{1} \mathrm{~N}$ Coelho, ${ }^{*} \mathrm{~A}$ P Pereira, ${ }^{2} \mathrm{D}$ Cunha, ${ }^{1} \mathrm{M}$ Theme. ${ }^{1}$ Oswaldo Cruz Foundation, Rio de Janeiro, Brazil; ${ }^{2}$ Federal University of Rio de Janeiro, Rio de Janeiro, Brazil

Objective To identify dietary patterns in pregnant women and investigate whether they are associated with sociodemographic factors, lifestyles, gestational diseases and pregestational body mass index.

Design and Methods Longitudinal study was carried out with 1482 pregnant women in two cities in the State of Rio de Janeiro, Brazil. A FFQ was applied retrospectively to assess diet at the third trimester of gestation. Principal components analysis was used to identify dietary patterns. Multiple linear regression model was used to study the associations between diet and covariates.

Results Four factors were identified: "Prudent" pattern (dairies, cracker, fruits and meat); "Traditional" (rice, beans, vegetables, bread, butter and sugar); "Snack" (salty snacks, sandwich cookies, and chocolate); "Western" (fast food, processed meat, eggs, sweet drinks, and other food with high carbohydrate content). The "Prudent" pattern was positively associated with maternal age $(B=0.012$, $\mathrm{p}=0.04)$, per capita family income $(B=0.253 \mathrm{p}<0.001)$ and negatively associated with pregestational overweigh $(B=-0.202, p=0.008)$ and gestational anaemia $(B=-0.060, p=0.04)$. The "Traditional" was inversely associated with gestational diabetes $(B=-0.551, p=0.002)$, city $(B=-0.189, p=0.002)$ and positively with smoking $(B=0.179$, $\mathrm{p}=0.038)$. The "Western" was inversely associated with instruction level $(B=-0.024, p=0.05)$, pregestational obesity $(B=-0.210$, $p=0.05)$ and positively with smoking $(B=0.187, p=0.05)$ and city $(\mathrm{B}=0.138, \mathrm{p}=0.04)$. The "Snack" pattern was positively associated with alcohol consumption $(B=0.274, p=0.01)$, high social class $(B=0.121, p=0.03)$ and inversely associated with maternal age $(\mathrm{B}=-0.044, \mathrm{p}<0.001)$.

Conclusion Four distinct dietary patters were identified which may be useful for further research concerning maternal diet and health outcomes among mothers and their offspring.

\section{P1-414 AGGRESSION BETWEEN INTIMATE PARTNERS IN NEW ZEALAND: GENDER DIFFERENCES AND ALCOHOL INVOLVEMENT}

doi:10.1136/jech.2011.142976g.4

${ }^{1} \mathrm{~J}$ Connor, ${ }^{*}{ }^{2} \mathrm{~K}$ Kypri, ${ }^{3} \mathrm{M}$ Bell, ${ }^{1} \mathrm{~K}$ Cousins. ${ }^{1}$ University of Otago, Dunedin, New Zealand; '2University of Newcastle, Newcastle, Australia; ${ }^{3}$ University of Sydney, Sydney, Australia

Introduction Higher rates of aggression have been described among intimate partners who are heavy drinkers. Drinking at the time of aggressive incidents, and gender differences in the experience of intimate partner aggression in the general population are less well understood.

Methods Alcohol consumption, partner's alcohol consumption, and details of the most severe incident of partner aggression in the past 2 years were reported in a national survey of $18-70$ year olds 
$(\mathrm{n}=1925)$. Mean levels of aggression, anger and fear were analysed by gender and alcohol involvement. Multinomial models estimated associations of drinking patterns with aggression to and from the respondent.

Results The response rate was 49\%. Men and women reported similar prevalence of victimisation and perpetration of aggression $(11 \%-15 \%)$. Alcohol was involved in more than a quarter of incidents, and reported more often by women than men, particularly male-only drinking when the respondent was victimised. Women reported more severity, anger and fear accompanying victimisation than men, and these scores differed significantly by involvement of alcohol. Heavy episodic drinking by respondents was associated with a threefold increase in victimisation involving alcohol, and doubling of perpetration of aggression involving alcohol.

Conclusion In a cross-section of households, "counts" of aggressive acts do not reflect the reality of gender differences. The frequency of heavy drinking episodes is associated with the occurrence of aggression involving alcohol within relationships, and the involvement of alcohol in an incident of aggression is associated with increased severity, fear and anger particularly for women.

\section{P1-415 SOCIOECONOMIC STATUS AND SURGICAL OUTCOME AFTER HYSTERECTOMY}

doi:10.1136/jech.2011.142976g.5

\begin{abstract}
${ }^{1,2} \mathrm{~S}$ Daugbjerg, ${ }^{*}{ }^{3} \mathrm{G}$ Cesaroni, ${ }^{2} \mathrm{~B}$ Ottesen, ${ }^{4} \mathrm{~F}$ Diderichsen, ${ }^{1} \mathrm{M}$ Osler. ${ }^{1}$ Research Centre for Prevention and Health, Glostrup University Hospital, Glostrup, Capital Region of Denmark, Denmark; ${ }^{2}$ Department of Gynaecology, Juliane Marie Centre, Rigshospitalet, Copenhagen, Denmark; ${ }^{3}$ Department of Epidemiology, Local Health Authority Rome E, Rome, Italy; ${ }^{4}$ Department of Social Medicine, Institute of Public Health, University of Copenhagen, Copenhagen, Denmark
\end{abstract}

Objective To examine the association between socioeconomic status (SES) and eight selected surgical outcomes after hysterectomy and asses the role of lifestyle, co-morbidity and clinical conditions on the relationship.

Methods All 22150 women registered with a benign elective hysterectomy code in the national Danish Hysterectomy Database (DHD) from 2004 to 2008 were included in the study. Data from DHD were linked to several central registers providing information on education, employment, income, lifestyle factors, co-morbidities and surgical outcome. Data were analysed using multilevel logistic regression models.

Results Overall $17 \%$ of the women experienced a clinical complication in relation to hysterectomy. Four per cent were re-operated, $6 \%$ readmitted and $6 \%$ experienced prolonged hospitalisation $\geq 5$ days. Women with short education had a higher risk of complications than women with higher education after adjusting for patient characteristics ( $O R=1.15,95 \%$ CI 1.02 to 1.31$)$. They also had a higher risk of peroperative bleeding after adjusting for lifestyle factors and comorbidity ( $\mathrm{OR}=1.60$, CI 1.15 to 2.22). Furthermore, a higher risk of infection and readmission was observed however, these relations seemed fully explained by lifestyle factors and co-morbidity. Women out of employment had a higher risk of infection, organ lesion, prolonged hospitalisation and readmission than women in employment. We found no association between income and surgical outcome after hysterectomy.

Conclusion This study suggests that women with low SES have a significant worse surgical outcome after hysterectomy than women with high SES when differences in lifestyle factors and co-morbidity are taking into account.

\section{P1-416 SECULAR TREND OF OVERWEIGHT AND OBESITY IN ADOLESCENTS OF HIGH SOCIOECONOMIC LEVEL FROM A DEVELOPED CITY IN THE SOUTHEAST OF BRAZIL}

doi:10.1136/jech.2011.142976g.6

${ }^{1} \mathrm{~S} D$ Castilho, ${ }^{1} \mathrm{C}$ A Bento, ${ }^{1} \mathrm{C}$ D Pinheiro, ${ }^{1} \mathrm{M}$ Cocetti, ${ }^{2} \mathrm{~A}$ de Azevedo Barros. ${ }^{*}{ }^{1}$ Faculty of Medicine-Puccamp, Campinas, São Paulo, Brazil; ${ }^{2}$ State University of Campinas Medical School, Campinas, São Paulo, Brazil

Introduction Although already of high prevalence, overweight and obesity are still increasing in many parts of the world. The objective was to evaluate the secular trend of overweight and obesity in adolescents of high socioeconomic level over a decade in a developed city of Brazil.

Method Two cross-sectional studies included 2825 students (1348 evaluated in 2001 and 1477 evaluated in 2010) of both genders and ages 7 to 18 years, from private schools in Campinas, SP, Brazil. The schools were randomly chosen. The first sample included 663 boys and 685 girls and the second included 709 boys and 763 girls. The BMI/age cut-off points of the Who Growth Standards 2007 were considered in the nutritional diagnosis: overweight $>+1 \mathrm{SD}$ and obesity $>+2$ SD. This study was approved by the Research Ethics Committee of PUC-Campinas (protocol no. 693/09).

Results The percentage of overweight adolescents (23\%) remained stable during this period. Obesity increased from 10\% (95\% CI 8.4 to $11.6)$ in 2001 to $15 \%$ (95\% CI 13.2 to 16.8 ) in 2010. Overweight and obesity were greater in boys than girls in both samples. Overweight and obesity were respectively $25 \%$ and $15 \%$ (95\% CI 12.3 to 17.7 ) in $2001,23 \%$ and $19 \%$ (95\% CI 16.1 to 21.9 ) in 2010 for boys, and $22 \%$ and $6 \%(95 \%$ CI 5.1 to 6.9$)$ in 2001 and $22 \%$ and $10 \%$ (95\% CI 7.9 to 12.1) in 2010 for girls.

Conclusion These data show that obesity are increasing and point to the need for immediate interventions in order to prevent this trend.

\section{P1-417 DOES LIFE-COURSE SOCIOECONOMIC POSITION EXPLAIN COLOUR/RACE INEQUALITIES IN THE PREVALENCE OF SELF- REPORTED UTERINE LEIOMYOMAS? EVIDENCE FROM THE PRÓ-SAÚDE STUDY, BRAZIL}

doi:10.1136/jech.2011.142976g.7

Karine de Lima Sírio Boclin, ${ }^{*}$ E Faerstein. Universidade do Estado do Rio de Janerio, Rio de Janeiro, RJ, Brazil

Introduction USA studies showed that uterine leiomyomas (UL) occur more frequently among black women, but the nature of this association remains largely unexplained. Because black women are disproportionately disadvantaged in social hierarchies, such racial inequality might be explained by life-course socioeconomic adversity. Aim To investigate whether life-course socioeconomic position (SEP) - during childhood, early adulthood, or lifelong-is a mediator of associations between colour/race and UL among Brazilian women. Methods We analysed data from self-administered questionnaires completed by 1534 female civil servants at a Rio de Janeiro university during the baseline data collection of the Pró-Saúde Study. Three study outcomes were explored: self-reported medical diagnosis of UL; UL with symptoms prior to diagnosis; and hysterectomy due to UL. The childhood SEP was assessed by parental education and the early adulthood SEP measured by participants' education; the cumulative SEP measures resulted from a combination of the previous information. We estimated ORs and 95\% CIs by logistic regression models.

Results Compared to whites, black and brown (mixed race) women presented risks respectively $1.6(95 \%$ CI 1.2 to 2.1$)$ and 1.4 (95\% CI 0.8 to 2.5$)$ higher of UL. This estimate was higher for those submitted to hysterectomy due to UL (blacks-OR: 2.6 to $1.7 ; 4.0$ 